\title{
Enhanced Gene Expression Following Vaccination in Chronic Fatigue Syndrome/Myalgic Encephalomyelitis
}

\author{
Ekua W. Brenu ${ }^{1,2 *}$, Gunn M. Atkinson ${ }^{2}$, Mieke L. van Driel ${ }^{3}$, Sanne Kreijkamp-Kaspers ${ }^{4}$, \\ Don R. Staines ${ }^{2,5}$, Sonya M. Marshall-Gradisnik ${ }^{1,2}$ \\ ${ }^{1}$ School of Medical Science, Griffith Health Institute, Griffith University, Gold Coast, Australia; ${ }^{2}$ National Center for Neuroimmu- \\ nology and Emerging Diseases, Griffith University, Gold Coast, Australia; ${ }^{3}$ Discipline of General Practice, School of Medicine, The \\ University of Queensland, Brisbane, Australia; ${ }^{4}$ Faculty of Health Sciences and Medicine, Bond University, Gold Coast, Australia; \\ ${ }^{5}$ Queensland Health, Gold Coast Public Health Unit, Gold Coast, Australia. \\ Email: *e.brenu@griffith.edu.au
}

Received December $4^{\text {th }}, 2012$; revised January $29^{\text {th }}, 2013$; accepted March $11^{\text {th }}, 2013$

\begin{abstract}
Vaccines have been shown to cause differential expression of genes and increase antibody titers against antigens. Influenza vaccines may have an effect on unexplained disorders such as Chronic Fatigue Syndrome/Myalgic Encephalomyelitis (CFS/ME). Immunological changes have been identified following immunization with trivalent influenza vaccine (TIV). The objective of this pilot study was to examine the consequences of TIV on cytokine and cytotoxic genes in CFS/ME. Peripheral blood mononuclear cells were preferentially isolated from whole blood of $7 \mathrm{CFS} / \mathrm{ME}$ patients and 8 controls. Following total RNA extraction and synthesis of cDNA, reverse transcriptase-quantitative polymerase chain reaction (RT-qPCR) was used to determine the expression levels of mRNAs for cytotoxic genes (perforin $(P R F 1)$, granzyme A $(G Z M A)$, granzyme $\mathrm{B}(G Z M B)$ and cytokine genes. GZMB was significantly increased overall in the CFS/ME patients compared to the controls. GZMA was significantly increased 28 days after vaccination while $P R F 1$ was reduced pre-vaccination but increased 14 days post-vaccination in the CFS/ME patients. There were no significant changes in cytokine genes pre or post vaccination. Administration of TIV may increase the expression of lytic genes in CFS/ME and this may contribute to the increase in cytotoxic activity we observed in these patients post vaccination.
\end{abstract}

Keywords: Chronic Fatigue Syndrome; Perforin; Granzymes; Cytokines

\section{Introduction}

The effectiveness of seasonal vaccines such as the influenza vaccine is related to the ability to establish antigenicity involving the type of vaccine generated and the strain of influenza vaccine present in the periphery [1]. Usually, the efficacy of a particular vaccine is conditional on intrinsic individual characteristic such as age, pre-existing antibody titers derived from vaccination or previous influenza infections and immune-competence of the individual [2]. Importantly, influenza vaccines are known to induce changes in the immune system in order to give a protective effect. In particular, trivalent inactivated influenza vaccine (TIV) can improve the titers of antibodies that are antigen specific. Similarly, both TIV and live attenuated influenza vaccine (LAIV) can stimulate changes in genes that are associated with the inter-

"Corresponding author. feron (IFN) signaling pathway including IRF7, IRF3, STAT1 and STAT2 [3]. These molecular changes may be cell specific. For example, in mice TIV vaccines induce changes in genes related to dendritic cells and B cells [4].

Administration of vaccines in patients with immunecompromised disorders is controversial, as there is a tendency for the vaccine to either exacerbate the symptoms or further suppress the already compromised immune system, thus causing detriment to health [5]. Importantly, it is difficult to predict the benefits of vaccination in people with diseases such as Chronic Fatigue Syndrome/Myalgic Encephalomyelitis (CFS/ME). CFS/ ME is largely a disease with unexplained mechanism(s) where a causal factor(s) currently remains unknown. Presently, the predominant method for identifying patients with CFS/ME is based on self-report tests [6]. Despite limitations in the diagnosis of CFS, impairments in immune function have been identified as important hall- 
marks of CFS/ME. Essentially, Natural Killer (NK) cell cytotoxic activity has been identified as an important immune deficit that may have biomarker potential in CFS/ME [7].

We have previously illustrated that administration of TIV may alter some immune indices in CFS/ME patients including cytotoxic activity, FOXP3 and the secretion of certain cytokines [8]. The exact mechanism for explaining these observed changes has not been identified and it is likely that in CFS/ME vaccination with TIV may affect molecular processes. Incidentally, gene expression studies have identified genes that may regulate immune, metabolic, endocrine and neurologic functions in CFS/ ME [9]. Hence, the aim of this pilot study was to explore the effects of vaccination with TIV on gene expression in patients with CFS/ME compared to non-fatigued controls.

\section{Method}

\subsection{Subject Recruitment}

Ethical approval was obtained from the Bond University Human Research Ethics Committee (R0852A). The study included CFS/ME participants $(\mathrm{n}=8$; age $=48$ years $)$ and non-fatigued controls $(\mathrm{n}=7$; age $=38$ years) [7]. The inclusion criteria for CFS/ME were based on the Centre for Disease Prevention and Control (CDC) 1994 case definition, while the non-fatigued controls were participants with no medical history or symptoms of prolonged fatigue or illness of any kind [10]. Patients were recruited into a larger study investigating potential biomarkers for CFS/ME in 2009 [11] and volunteers were invited to participate in this pilot study before the influenza season in May 2011. All participants were screened by a general practitioner on site for contra-indications to vaccination as per the Australian immunization handbook [12]. Participants provided written consent and filled out a brief survey. A qualified medical doctor administered the TIV immunization, Influvac (Solvay Pharmaceuticals, Sydney, NSW). A volume of $0.5 \mathrm{~mL}$ in a pre-filled syringe containing $15 \mu \mathrm{g}$ haemagglutinin of each of the three recommended strains produced for the 2011 flu season ((2011) A/California/, 7/2009 (H1N1)-like strain, A/ Perth/, 16/2009 (H3N2)-like strain and B/, Brisbane/60/ 2008-like strain) was used. The vaccine was administered intramuscularly to the deltoid muscle.

\subsection{Sample Collection}

Venous blood samples $(40 \mathrm{~mL})$ drawn from all participants were collected into EDTA tubes and analysed within three hours of collection. Peripheral blood mononuclear cells (PBMCs) were isolated from whole blood using Ficoll-hypaque (GE Healthcare, Uppsala, Sweden). Samples were collected prior to vaccination and 14 and
28 days post vaccination.

\subsection{RNA Extraction and cDNA Synthesis}

Total RNA was extracted from PBMCs using the miRNeasy isolation kit (Qiagen, Hilden, Germany) according to manufacturer's instructions. Concentration and purity of RNA was determined using the NanoDrop 3300 (Thermo Scientific, Waltham, MA). Following RNA extraction and quantification, synthesis of RNA to cDNA was accomplished with the SuperScript ${ }^{\mathrm{TM}}$ III First-Strand synthesis SuperMix for reverse transcriptase-quantitative polymerase chain reaction (RT-qPCR) (Invitrogen, Carlsbad, CA) according to the manufacturer's instructions. Synthesized cDNA were diluted 1:20 and stored at $-20^{\circ} \mathrm{C}$ prior to RT-qPCR.

\subsection{RT-qPCR Analysis}

RT-qPCR was performed in a CFX96 Real-Time PCR Detection System (Bio-Rad, Hercules, CA). The final reaction volume $(10 \mu \mathrm{L})$ included $1 \times$ iQ SYBR-Green Supermix (Bio-Rad, Hercules, CA), $200 \mathrm{nM}$ of each primer and $4 \mu \mathrm{L}$ of diluted cDNA. The RT-qPCR conditions were $95^{\circ} \mathrm{C}$ for 3 minutes, followed by 45 cycles of $95^{\circ} \mathrm{C}$ for $10 \mathrm{~s}, 62^{\circ} \mathrm{C}$ for $30 \mathrm{~s}$ and generation of melt curves at $65^{\circ} \mathrm{C}$ to $95^{\circ} \mathrm{C}$ for the detection of secondary amplicons [13]. All reactions were performed in triplicate with each reaction plate containing an equal number of $\mathrm{CFS} / \mathrm{ME}$ and non-fatigued controls, a calibrator control derived from a pool of all cDNA samples and a no template control. PCR amplification efficiencies $(85 \%$ $100 \%$ ) for each primer pair was calculated using a 4-log serial dilution of the calibrator sample and efficiency correction was applied to the data during analysis.

\subsection{Stability of Normalization Gene}

Determination of the most stable mRNA gene for normalization purposes was performed on the bio-rad CFX manager analysis software which allowed the comparison of $\mathrm{M}$-values for each of the housekeeping genes. GAPDH, PGK1, $18 \mathrm{~S}$ and ACTIN- $\beta$ were the housekeeping genes tested for stability. M-values greater than 1 were rejected while an M-value less than 1 for a particular gene was considered the most stable.

\subsection{Statistical Analysis}

The data generated from the PCR were evaluated using the CFX Manager v1.6 (Bio-Rad, Hercules, CA). Interassay variations were normalized using a calibrator sample. Normalized expression ( $\Delta \Delta \mathrm{Cq})$ for the genes of interest was normalized to a calibrator control and reference gene GAPDH. All values for expression were $\log _{2}$ transformed. The results were analysed using GraphPad 
Prism 5 (GraphPad Software, San Diego, CA). Results were statistically significant where $\mathrm{P} \leq 0.05$. Non-parametric Mann-Whitney test was used in analysing all data.

\section{Results}

\subsection{RT-qPCR for Reference Gene Selection}

As the stability of a reference gene is paramount for accurate outcomes in gene expression studies, a number of reference genes were inspected to establish the best possible gene for this study. GAPDH was found to be the most stable of the other housekeeping genes PGK1, 18S and ACTIN- $\beta$. GAPDH was determined to be the most stable reference gene as its average expression was less than one (Figure 1).

\subsection{RT-qPCR for Lytic and Cytokine Genes}

The expression levels of 6 genes were examined in the CFS/ME patients and non-fatigued controls. These included mRNA levels for lytic genes, PRF1, GZMA and GZMB, and cytokine genes IFN-G, TGF-B and TNF-A. In comparison to the non-fatigued controls, the CFS/ME patients, lytic proteins were significantly altered before and after vaccination. PRF1 was the only gene that was significantly decreased in the CFS/ME patients prior to vaccination (Figure 2). However, PRF1 was increased 14 days after vaccination while GZMA was increased 28

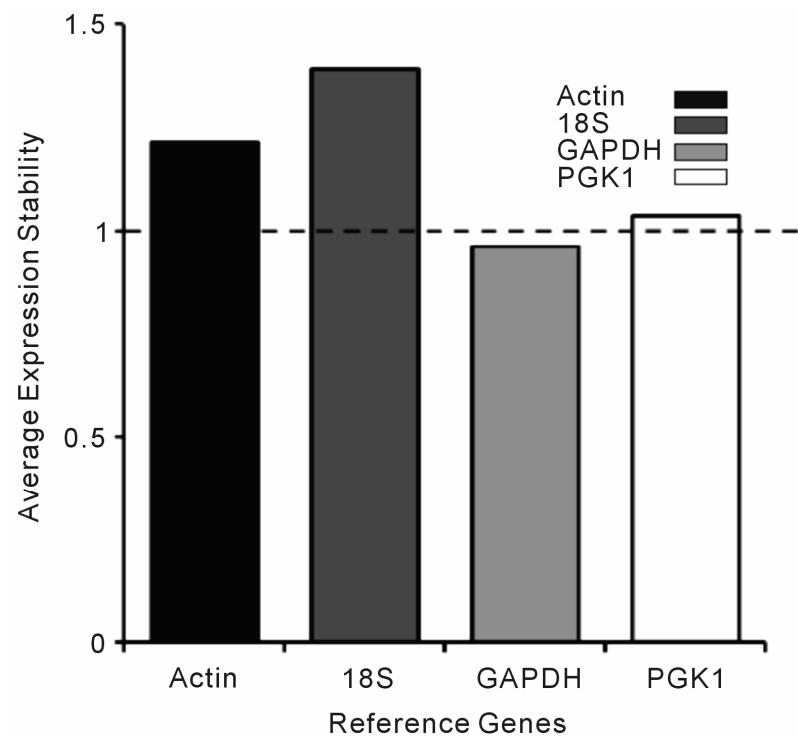

Figure 1. Determination of the appropriate reference gene for gene expression based on M-values (average expression). The stability of the reference genes (Actin, 18S, GAPDH and PGK1) was examined to determine the most stable reference gene suitable for this study. Each bar in the graph above represents the average expression of all participants for a particular reference gene. The dashed line represents the threshold value (1.0) using the CFX manger analysis software.

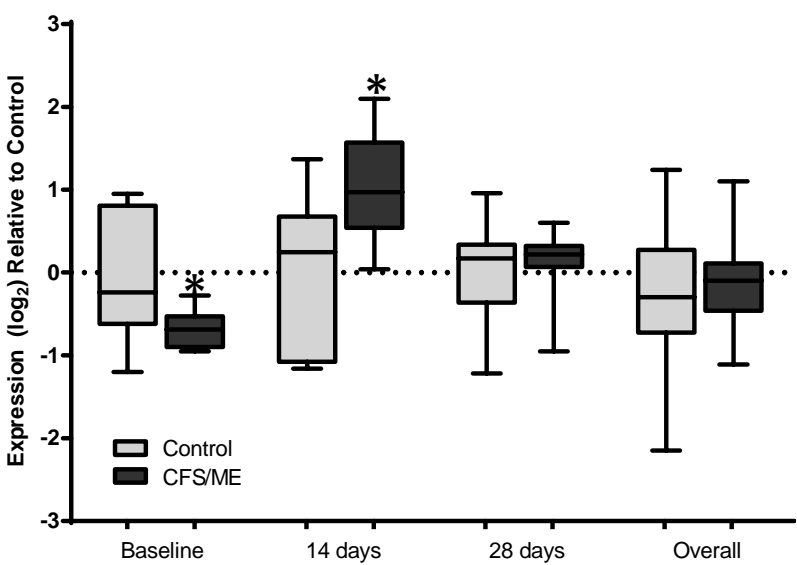

Figure 2. Analysis of $P R F 1$ expression pre- and post-vaccination. The expression profile of $P R F 1$ at baseline prior to vaccination, 14 and 28 days post-vaccination and the overall expression of these genes following a pooled analysis of all time points is presented. The grey box plots are representative of control data while the black box plots represent CFS/ME data. The boxes are indicative of the interquartile range $(25 \%-75 \%)$, with the median illustrated by the presence of the horizontal bars within the boxes and the whiskers below and above are symbolic of the minimum and maximum data sets. ${ }^{*}$-values $\leq 0.05$.

days after vaccination in the CFS/ME group (Figures 2 and 3). An overall increase in GZMB was noticed in the pooled CFS/ME patients sample in comparison to the non-fatigued controls (Figure 4). Cytokine genes were not significantly different between groups prior or post vaccination (data not shown).

\section{Discussion}

The results from this pilot investigation suggest that TIV may enhance the expression of lytic genes in patients with immune-compromised disorders such as CFS/ME. Prior to vaccination there was a trend towards reduced mean fold in $P R F 1$ expression in the CFS/ME patients compared to the non-fatigued controls. Both granzyme genes were similar in expression in the two groups. However, $P R F 1$ and GZMA were significantly increased 14 days and 28 days after vaccination, respectively. There were no observable changes in cytokine expression prior or post vaccination.

Previous reports have provided evidence in support of molecular changes following vaccination. In most of these studies, an upregulation in immune related genes has been observed in healthy individuals after vaccination. The pattern of response and the effects on molecular processes are time dependent and most often extreme expression changes have been observed in the first 24 hours following vaccination [13].

$P R F 1, G Z M A$ and $G Z M B$ are translated in to perforin and granzyme proteins which are predominantly found 


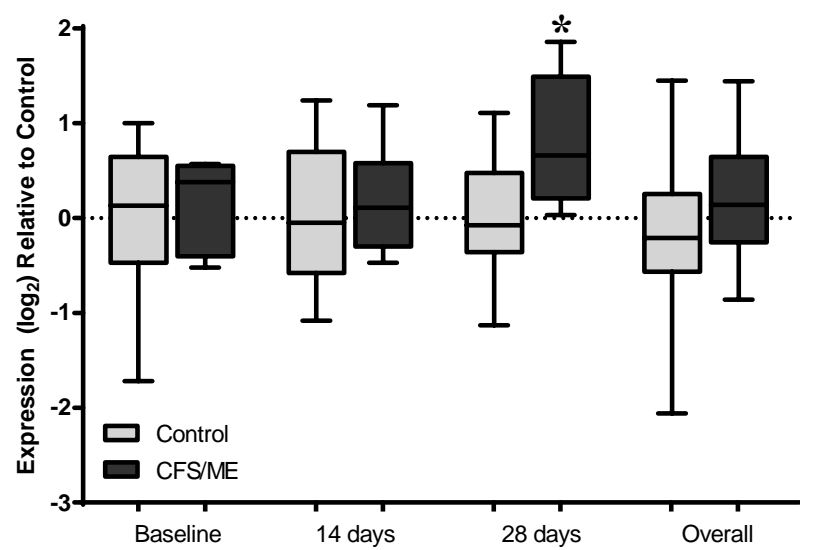

Figure 3. Analysis of GZMA expression pre- and post-vaccination. The expression profile of GZMA at baseline prior to vaccination, 14 and 28 days post-vaccination and the overall expression of these genes following a pooled analysis of all time points is presented. The grey box plots are representative of control data while the black box plots represent CFS/ME data. The boxes are indicative of the interquartile range $(25 \%-75 \%)$, with the median illustrated by the presence of the horizontal bars within the boxes and the whiskers below and above are symbolic of the minimum and maximum data sets. "P-values $\leq \mathbf{0 . 0 5}$.

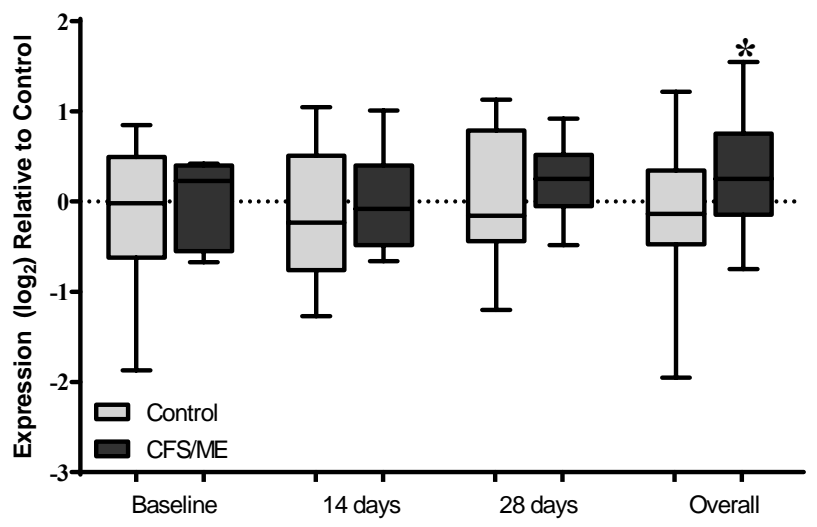

Figure 4. Analysis of GZMB expression pre- and post-vaccination. The expression profile of GZMB at baseline prior to vaccination, 14 and 28 days post-vaccination and the overall expression of these genes following a pooled analysis of all time points is presented. The grey box plots are representative of control data while the black box plots represent CFS/ME data. The boxes are indicative of the interquartile range $(25 \%-75 \%)$, with the median illustrated by the presence of the horizontal bars within the boxes and the whiskers below and above are symbolic of the minimum and maximum data sets. ${ }^{*}$-values $\leq \mathbf{0 . 0 5}$.

in cytotoxic lymphocytes. These proteins are used to induce cellular apoptosis of viral infected cells $[14,15]$. During cytotoxic activity against viral infection, perforin forms pores in the cell membrane via a calcium dependent pathway enabling the release and delivery of granzymes into the target cell where they induce apoptosis [16-18].
In the present study influenza vaccine enhanced the expression of $P R F 1$ and this may be consistent with the finding that cytotoxic activity increased significantly 14 days after vaccination in the CFS/ME patients compared to the non-fatigued controls [8]. IL-2R has been observed to heighten $P R F 1$ expression particularly in $\mathrm{CD} 8^{+} \mathrm{T}$ cells and reductions in IL-2R dampen the expression of $P R F 1$ $[19,20]$. A similar observation has been reported in NK cells in the presence of IL-2 [21,22]. In NK cells IL-2R $\beta$ regulates the concentration of perforin [23] via Janus Kinase/STAT or the $\mathrm{NF} \kappa \mathrm{B}$ pathway. The JAK/STAT pathway results in the activation of STAT5 [19]. IL-2R signalling activates IKK $\alpha$ which results in the recruitment of $\mathrm{I} \kappa \mathrm{B} \alpha$ and this stimulates the binding of $\mathrm{NF} \kappa \mathrm{B}$ components resulting in an enhanced $P R F 1$ expression in NK cells [22]. Relatively low levels of IL-2 are present in the plasma during influenza infection causing a possible reduction in NK related IFN- $\gamma$ secretion and cytotoxic activity [24]. Reduced cytotoxic activity is a hallmark of CFS/ME and this may promote viral thrive, persistence and suppressed pathogen clearance. Influenza vaccines such as LIV have been reported to increase the production of IL-2 $[25,26]$. Thus, it is possible that administration of TIV provoked an excess in IL- 2 or IL-2R consequently stimulating a significant increase in $P R F 1$ and cytotoxic activity. During influenza virus infection, cytotoxic cells in the respiratory tract lyse infected cells in the mucosal membrane, using the perforin pathway [27]. This is an important early response to influenza and ensures rapid clearance of the viral pathogens.

The exact cause for the increase in GZMA after vaccination is currently unknown however, GZMA is known to promote pro-inflammatory cytokine release, hence the increase in GZMA may have induced significant increases in pro-inflammatory cytokines including TNF- $\alpha$ [28]. GZMA and GZMB have been shown to be decreased in CFS/ME patients [7,29]. GZMB is an important marker of susceptibility to influenza in older adults [30]. Although, GZMB was markedly increased in the CFS/ME patients 14 days post-vaccination, it was not statistically significant. Similarly, cytokine mRNA expressions were not significantly changed in the present study. Conversely in animal experiments, influenza increases IL-4, IL-5, IL-6 and IL-10 mRNA levels two days post vaccination [31], highlighting a potential influence of time on changes in mRNA levels. Hence, measurement of expression changes at immediate time intervals maybe necessary for future studies as salient effects potentially occur immediately following vaccination.

At the molecular level vaccination against influenza may have a significant influence on the expression of immune related genes at different time points. These effects occur only in the short run and do not persist over- 
time. Therefore at the initial stages of vaccine introduction in CFS/ME, these effects may be beneficial as they may cause a significant improvement in mechanisms such as cytotoxic activity that are observed to be decreased in CFS/ME. Hence, vaccination may be protective in the short run causing an improvement in immune function. Thus, it can be postulated that the increase in cytotoxic activity in the CFS/ME patients occurred as a consequence of influenza vaccination, leading to an increase in PRF1 and a subsequent increase in cytotoxic activity.

The limitations with this study pertain to the sample size, as a larger sample size may substantiate the results. As this was a pilot study, further investigations are now required using a large sample size and a broad spectrum of genes. This study has demonstrated salient changes in cytotoxic-related genes that may correlate with the functional status of $\mathrm{NK}$ cells in $\mathrm{CFS} / \mathrm{ME}$ patients following vaccination. Moreover, whether these effects are detrimental to CFS/ME immune health is yet to be proven as changes in lytic genes did not persist for the duration of the study.

\section{REFERENCES}

[1] P. R. Dormitzer, G. Galli, F. Castellino, H. Golding, S. Khurana, G. Del Giudice and R. Rappuoli, "Influenza Vaccine Immunology," Immunological Reviews, Vol. 239, No. 1, 2011, pp. 167-177. doi:10.1111/j.1600-065X.2010.00974.x

[2] N. E. Basta, M. E. Halloran, L. Matrajt and I. M. Longini Jr., "Estimating Influenza Vaccine Efficacy from Challenge and Community-Based Study Data," American Journal of Epidemiology, Vol. 168, No. 12, 2008, pp. 13431352. doi:10.1093/aje/kwn259

[3] H. I. Nakaya, J. Wrammert, E. K. Lee, L. Racioppi, S. Marie-Kunze, W. N. Haining, A. R. Means, S. P. Kasturi, N. Khan, G. M. Li, M. McCausland, V. Kanchan, K. E. Kokko, S. Li, R. Elbein, A. K. Mehta, A. Aderem, K. Subbarao, R. Ahmed and B. Pulendran, "Systems Biology of Vaccination for Seasonal Influenza in Humans," Nature Immunology, Vol. 12, 2011, pp. 786-795. doi:10.1038/ni.2067

[4] C. A. Siegrist, "Vaccinology Update for 2008: What's New in Switzerland?" Revue Médicale Suisse, Vol. 4, No. 139, 2008, pp. 72-75.

[5] M. Bijl, C. G. Kallenberg and S. van Assen, "Vaccination of the Immune-Compromised Patients with Focus on Patients with Autoimmune-Inflammatory Diseases," The Netherlands Journal of Medicine, Vol. 69, No. 1, 2011, pp. 5-13.

[6] W. C. Reeves, D. Wagner, R. Nisenbaum, J. F. Jones, B. Gurbaxani, L. Solomon, D. A. Papanicolaou, E. R. Unger, S. D. Vernon and C. Heim, "Chronic Fatigue Syndrome-A Clinically Empirical Approach to Its Definition and Study," BMC Medicine, Vol. 3, 2005, p. 19. doi:10.1186/1741-7015-3-19
[7] E. W. Brenu, M. L. van Driel, D. R. Staines, K. J. Ashton, S. B. Ramos, J. Keane, N. G. Klimas and S. M. Marshall-Gradisnik, "Immunological Abnormalities as Potential Biomarkers in Chronic Fatigue Syndrome/Myalgic Encephalomyelitis," Journal of Translational Medicine, Vol. 9, 2011, p. 81. doi:10.1186/1479-5876-9-81

[8] E. W. Brenu, M. van Driel, D. R. Staines, S. KreijkampKaspers, S. L. Hardcastle and S. Marshall-Gradisnik, "The Effects of Influenza Vaccination on Immune Function in Patients with Chronic Fatigue Syndrome/ Myalgic Encephalomyelitis," International Journal of Clinical Medicine, Vol. 3, No. 6, 2012, pp. 544-551.

[9] J. R. Kerr, R. Petty, B. Burke, J. Gough, D. Fear, L. I. Sinclair, D. L. Mattey, S. C. Richards, J. Montgomery, D. A. Baldwin, P. Kellam, T. J. Harrison, G. E. Griffin, J. Main, D. Enlander, D. J. Nutt and S. T. Holgate, "Gene Expression Subtypes in Patients with Chronic Fatigue Syndrome/Myalgic Encephalomyelitis," The Journal of Infectious Diseases, Vol. 197, No. 8, 2008, pp. 11711184. doi: $10.1086 / 533453$

[10] K. Fukuda, S. E. Straus, I. Hickie, M. C. Sharpe, J. G. Dobbins and A. Komaroff, "The Chronic Fatigue Syndrome: A Comprehensive Approach to Its Definition and Study. International Chronic Fatigue Syndrome Study Group," Annals of Internal Medicine, Vol. 121, No. 12, 1994, pp. 953-959.

[11] E. W. Brenu, D. R. Staines, O. K. Baskurt, K. J. Ashton, S. B. Ramos, R. M. Christy and S. M. Marshall-Gradisnik, "Immune and Hemorheological Changes in Chronic Fatigue Syndrome," Journal of Translational Medicine, Vol. 8, 2010, p. 1. doi:10.1186/1479-5876-8-1

[12] "The Australian Immunisation Handbook," 9th Edition, 2008.

http://www.health.gov.au/internet/immunise/publishing.n sf/Content/Handbook-influenza

[13] K. L. Bucasas, L. M. Franco, C. A. Shaw, M. S. Bray, J. M. Wells, D. Nino, N. Arden, J. M. Quarles, R. B. Couch and J. W. Belmont, "Early Patterns of Gene Expression Correlate with the Humoral Immune Response to Influenza Vaccination in Humans," Journal of Infectious Diseases, Vol. 203, No. 7, 2011, pp. 921-929. doi:10.1093/infdis/jiq156

[14] J. Stein-Streilein, M. Bennett, D. Mann and V. Kumar, "Natural Killer Cells in Mouse Lung: Surface Phenotype, Target Preference, and Response to Local Influenza Virus Infection," Journal of Immunology, Vol. 131, No. 6, 1983, pp. 2699-2704.

[15] P. C. Doherty, D. J. Topham, R. A. Tripp, R. D. Cardin, J. W. Brooks and P. G. Stevenson, "Effector CD4+ and CD8+ T-Cell Mechanisms in the Control of Respiratory Virus Infections," Immunological Reviews, Vol. 159, No. 1, 1997, pp. 105-117. doi:10.1111/j.1600-065X.1997.tb01010.x

[16] M. G. Lichtenheld, K. J. Olsen, P. Lu, D. M. Lowrey, A. Hameed, H. Hengartner and E. R. Podack, "Structure and Function of Human Perforin," Nature, Vol. 335, 1988, pp. 448-451. doi:10.1038/335448a0

[17] M. G. Lichtenheld and E. R. Podack, "Structure of the Human Perforin Gene. A Simple Gene Organization with 
Interesting Potential Regulatory Sequences," Journal of Immunology, Vol. 143, No. 12, 1989, pp. 4267-4274.

[18] S. Hoves, J. A. Trapani and I. Voskoboinik, "The Battlefield of Perforin/Granzyme Cell Death Pathways," Journal of Leukocyte Biology, Vol. 87, No. 2, 2010, pp. 237243. doi:10.1189/jlb.0909608

[19] J. Zhang, I. Scordi, M. J. Smyth and M. G. Lichtenheld, "Interleukin 2 Receptor Signaling Regulates the Perforin Gene through Signal Transducer and Activator of Transcription (Stat)5 Activation of Two Enhancers," Journal of Experimental Medicine, Vol. 190, No. 9, 1999, pp. 1297-1308. doi:10.1084/jem.190.9.1297

[20] M. E. Pipkin, A. Rao and M. G. Lichtenheld, "The Transcriptional Control of the Perforin Locus," Immunological Reviews, Vol. 235, No. 1, 2010, pp. 55-72.

[21] Y. F. Wu, B. H. Zhang, D. Y. Cen, J. Wei and C. Chen, "Expression of Perforin in Cord Blood NK Cells after IL-2/IL-15 Stimulation and Its Relation with Cytotoxicity," Journal of Experimental Hematology, Vol. 19, No. 4, 2011, pp. 1015-1018.

[22] J. Zhou, J. Zhang, M. G. Lichtenheld and G. G. Meadows, "A Role for NF-Kappa B Activation in Perforin Expression of NK Cells upon IL-2 Receptor Signaling," Journal of Immunology, Vol. 169, No. 3, 2002, pp. 1319-1325.

[23] T. W. Salcedo, L. Azzoni, S. F. Wolf and B. Perussia, "Modulation of Perforin and Granzyme Messenger RNA Expression in Human Natural Killer Cells," Journal of Immunology, Vol. 151, No. 5, 1993, pp. 2511-2520.

[24] S. Jost, H. Quillay, J. Reardon, E. Peterson, R. P. Simmons, B. A. Parry, N. N. Bryant, W. D. Binder and M. Altfeld, "Changes in Cytokine Levels and NK Cell Activation Associated with Influenza," PLoS One, Vol. 6, No. 9, 2011, Article ID: e25060. doi:10.1371/journal.pone.0025060

[25] A. N. Naikhin, A. R. Rekstin, I. B. Barantseva, S. A. Donina, A. Desheva Iu, E. P. Grigor'eva, I. V. Kiseleva and L. G. Rudenko, "Immune Response to Live Influenza Vaccine," Vestnik Rossiiskoi Akademii Meditsinskikh Nauk, Vol. 12, 2002, pp. 24-28.

[26] A. N. Naikhin, A. R. Rekstin, J. Katz, S. A. Donina, E. P. Grigor'eva, A. Desheva Iu, N. Arden, L. G. Rudenko and N. Cox, "Production of Interleukin-2 in Vitro and in Vivo in Elderly People, Vaccinated with Live and Inactivated Flu Vaccines Separately and Combined," Voprosy Virusologii, Vol. 45, No. 1, 2000, pp. 25-29.

[27] B. Liu, I. Mori, M. J. Hossain, L. Dong, Z. Chen and Y. Kimura, "Local Immune Responses to Influenza Virus Infection in Mice with a Targeted Disruption of Perforin Gene," Microbial Pathogenesis, Vol. 34, No. 4, 2003, pp. 161-167. doi:10.1016/S0882-4010(02)00191-2

[28] J. Pardo, J. I. Aguilo, A. Anel, P. Martin, L. Joeckel, C. Borner, R. Wallich, A. Mullbacher, C. J. Froelich and M. M. Simon, "The Biology of Cytotoxic Cell Granule Exocytosis Pathway: Granzymes Have Evolved to Induce Cell Death and Inflammation," Microbes and Infection, Vol. 11, No. 4, 2009, pp. 452-459. doi:10.1016/j.micinf.2009.02.004

[29] T. Saiki, T. Kawai, K. Morita, M. Ohta, T. Saito, K. Rokutan and N. Ban, "Identification of Marker Genes for Differential Diagnosis of Chronic Fatigue Syndrome," Molecular Medicine, Vol. 14, No. 9-10, 2008, pp. 599607. doi:10.2119/2007-00059.Saiki

[30] J. E. McElhaney, S. Gravenstein, C. M. Upshaw, J. W. Hooton, P. Krause, P. Drinka and R. C. Bleackley, "Granzyme B: A Marker of Risk for Influenza in Institutionalized Older Adults," Vaccine, Vol. 19, No. 27, 2001, pp. 3744-3751. doi:10.1016/S0264-410X(01)00087-1

[31] E. Szyszko, K. Brokstad, R. J. Cox, A. O. Hovden, A. Madhun and L. R. Haaheim, "Impact of Influenza Vaccine Formulation with a Detailed Analysis of the Cytokine Response," Scandinavian Journal of Immunology, Vol. 64, No. 5, 2006, pp. 467-475. doi:10.1111/j.1365-3083.2006.01805.x 